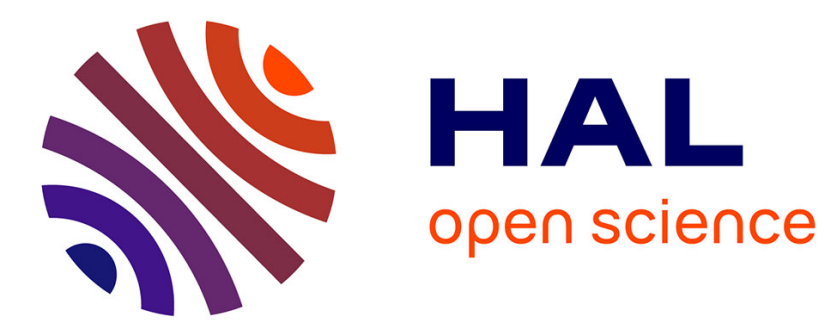

\title{
LA CRISE GRECQUE: UN SCANDALE MANQUÉ
} Jeremy Morales, Yves Gendron, Henri Guenin-Paracini

\section{To cite this version:}

Jeremy Morales, Yves Gendron, Henri Guenin-Paracini. LA CRISE GRECQUE : UN SCANDALE MANQUÉ. Comptabilités et innovation, May 2012, Grenoble, France. pp.cd-rom. hal-00690951

\section{HAL Id: hal-00690951 https://hal.science/hal-00690951}

Submitted on 24 Apr 2012

HAL is a multi-disciplinary open access archive for the deposit and dissemination of scientific research documents, whether they are published or not. The documents may come from teaching and research institutions in France or abroad, or from public or private research centers.
L'archive ouverte pluridisciplinaire HAL, est destinée au dépôt et à la diffusion de documents scientifiques de niveau recherche, publiés ou non, émanant des établissements d'enseignement et de recherche français ou étrangers, des laboratoires publics ou privés. 


\title{
LA CRISE GRECQUE : UN SCANDALE MANQUÉ
}

\author{
THE GREEK CRISIS : A MISSED SCANDAL
}

\section{Jérémy Morales*, Yves Gendron** et Henri Guénin-Paracini**}

\begin{tabular}{|c|c|}
\hline Résumé & Abstract \\
\hline $\begin{array}{l}\text { Cet article s'intéresse au renforcement de l'agenda } \\
\text { néolibéral de managérialisation des États dans le } \\
\text { contexte de crises qui pourraient, en principe, mettre à } \\
\text { l'épreuve sa légitimité. À travers le cas du maquillage } \\
\text { des statistiques publiques de l'État grec, nous étudions } \\
\text { comment les trajectoires problématisantes médiatiques } \\
\text { participent à la naturalisation du néolibéralisme en } \\
\text { produisant une interprétation collective favorable à la } \\
\text { perpétuation de l'ordre établi. }\end{array}$ & $\begin{array}{l}\text { This paper studies the reinforcement of state } \\
\text { managerialism in a context of potentially destabilising } \\
\text { crises. Through the case of the Greek making-up of its } \\
\text { public accounts, we analyse problematizing } \\
\text { trajectories followed in the media and that participate } \\
\text { in the naturalisation of neoliberalism by producing a } \\
\text { collective interpretation favouring the perpetuation of } \\
\text { the status quo. }\end{array}$ \\
\hline $\begin{array}{l}\text { Mots clés : Crise grecque, néolibéralisme, trajectoires } \\
\text { problématisantes }\end{array}$ & $\begin{array}{l}\text { Key words: } \begin{array}{c}\text { Greek } \\
\text { problematizing trajectories }\end{array}\end{array}$ \\
\hline
\end{tabular}

* ESCP Europe, CPO, 79, avenue de la République, 75543 Paris Cedex 11, France, jmorales@escpeurope.eu

** Université Laval, Faculté des Sciences de 1'Administration, Pavillon Palasis-Prince, 2325 rue de la Terrasse, Québec (QC), Canada, G1V 0A6, yves.gendron@fsa.ulaval.ca et henri.guenin-paracini@fsa.ulaval.ca 


\section{Introduction}

Au début de 1'année 2010, le Spiegel en Allemagne (le 8 février) puis le New York Times aux États-Unis (le 14 février) dénoncent : le gouvernement Grec a «maquillé »ses comptes. Rapidement, les journalistes se focalisent sur les questions qui leur semblent les plus importantes : les gouvernants des autres pays européens vont-ils accepter de prêter de l'argent au gouvernement grec ? La «crise grecque » va-t-elle se propager à d'autres pays européens, voire toucher l'ensemble des économies occidentales ? Cependant, cet épisode soulève une question qui nous semble plus significative. Gérés de plus en plus comme des entreprises, les gouvernements centraux semblent en imiter certaines techniques qui pourraient sembler peu intuitives pour une entité ne visant pas, a priori, la réalisation de profits. Doit-on alors imaginer que les États puissent s'inspirer des pratiques du privé au point de se lancer dans des montages financiers relevant de la comptabilité créative ?

Cet article s'intéresse aux mécanismes sociaux qui participent à la consolidation de l'agenda néolibéral de managérialisation des États, en dépit de crises qui pourraient, du moins théoriquement, mettre à l'épreuve sa légitimité. En mettant l'accent sur le cas du maquillage des statistiques publiques de l'État grec, nous cherchons à mieux comprendre comment un événement potentiellement déstabilisateur est problématisé, dans la presse écrite, de sorte qu'il ne remette pas en cause l'ordre établi.

La plupart des études du néolibéralisme le définissent comme un projet économique, politique et social fondé sur une diminution du rôle de l'État et promouvant les privatisations, les déréglementations et le libre-échange de manière à favoriser la généralisation des mécanismes de marché (Bourdieu, 1998; Duménil et Lévy, 2000 ; Tabb, 2003 ; Harvey, 2005 ; Soederberg, 2008). Selon cette définition, le néolibéralisme est une nouvelle forme de libéralisme apparue aux États-Unis et au Royaume-Uni dans les années 1980, puis promue et exportée par le Fonds Monétaire International (FMI), la Banque Mondiale et l'Organisation Mondiale du Commerce : ce serait l'idéologie qui a donné naissance, à la fin des années 1980, au «consensus de Washington » visant l'instauration d'une panoplie de mesures pour que les économies gouvernementales en difficulté puissent juguler « efficacement » leurs problèmes économiques. Les auteurs qui défendent ce point de vue montrent alors que les pratiques du néolibéralisme se sont éloignées de son projet initial pour favoriser l'intervention étatique (par exemple lors du soutien accordé régulièrement par les États ou par le FMI aux institutions financières en cas de crise économique), ce qu'ils considèrent comme une évolution paradoxale. Au contraire, Foucault (2004) définit le néolibéralisme comme un projet apparu dans les années 1940, très différent du libéralisme puisque le néolibéralisme est fondé sur une certaine forme d'intervention étatique. Les marchés ne sont plus une limite à l'intervention de l'État mais un objectif et un principe d'organisation et d'évaluation des politiques publiques : l'État doit construire un cadre social et politique favorable à l'émergence d'une société d'entrepreneurs placés en situation de concurrence. Cette vision a été prolongée par Dardot et Laval (2009) qui montrent les liens entre le projet néolibéral et la nouvelle gestion publique (voir également Broadbent et Guthrie, 1992 ; Hood, 1995 ; Guthrie et al., 2005). 
Notre article contribue à ce courant de recherche en montrant comment les mécanismes de problématisation médiatique participent à la naturalisation de la tendance néolibérale et permettent au managérialisme de sortir renforcé de ses mises à l'épreuve. Nous ne cherchons pas ici à dénoncer ou à condamner certains acteurs spécifiques, et nous ne présentons pas nos résultats comme révélant l'action concertée d'acteurs politiques, économiques ou médiatiques: nous cherchons plutôt à saisir les effets généraux de la manière dont un événement, potentiellement déstabilisateur, a été intégré à une interprétation spécifique ne remettant pas en cause la tendance néolibérale.

Les matériaux empiriques que nous mobilisons sont constitués d'énoncés journalistiques produits par la presse écrite de langue anglaise. Nous n'avons pas cherché à obtenir une documentation exhaustive en la matière, mais à compiler les données facilement accessibles à tout citoyen (comprenant l'anglais) qui aurait été intéressé par ce sujet. Pour cela, nous avons utilisé la base de données Factiva. La constitution de la documentation a été relativement pragmatique, au sens où plusieurs requêtes ont été lancées jusqu'à ce que nous ayons le sentiment d'avoir obtenu une image relativement complète des principaux thèmes abordés et des opinions émises, un peu à l'instar du principe de saturation. Nous avons aussi étudié les documents cités comme étant des rapports d'expert ou des documents officiels publiés sur le sujet.

Nous n'utilisons pas ces énoncés comme des sources d'information sur la situation grecque en tant que telle, mais comme un matériau permettant de mettre au jour les problématisations construites dans la presse écrite. Notre objectif était de retracer les mises en accusation et solutions proposées au «cas grec». Les journalistes produisent des interprétations quotidiennes et qui évoluent rapidement, ce qui nous a permis de mettre en lumière certaines «trajectoires problématisantes ». Notre approche ne se voulait donc pas systématique puisque notre intérêt était de repérer certaines tendances globales dans la problématisation médiatique de la crise grecque, et non pas d'en saisir toutes les nuances ${ }^{1}$.

Nous cherchons à comprendre comment certains modes de gouvernement voient leur légitimité renforcée par des mécanismes de mise à l'épreuve. Pour cela, nous retraçons ce que nous nommons des trajectoires naturalisantes de problématisation, à travers lesquelles certains phénomènes sont problématisés de sorte qu'ils ne remettent pas trop fortement en cause l'ordre établi. Notre analyse s'inspire notamment des perspectives critiques de recherche, appliquées en l'occurrence au « néolibéralisme».

Les approches critiques connaissent des influences diverses, et parfois contradictoires, ce qui rend délicate la définition d'un «courant critique » homogène. Cependant, plusieurs éléments communs aux approches critiques et les distinguant de recherches plus « conventionnelles » ont été identifiés (Fournier et Grey, 2000 ; Allard-Poesi et Loilier, 2009 ; Golsorkhi et al., 2009). Au-delà de la critique scientifique (scepticisme méthodologique visant à dépasser le sens commun), la posture critique désigne une réflexivité plus radicale (ce qui l'amène à abandonner toute illusion quant à la neutralité du chercheur), orientée vers le questionnement de ce qui est perçu comme nécessaire ou évident («dénaturaliser » les

\footnotetext{
${ }^{1}$ Les contraintes éditoriales nous interdisent de citer les sources de manière exhaustive : nous ne faisons donc apparaître que les références dont une partie du contenu est reproduite. Une liste complète est disponible sur demande aux auteurs.
} 
phénomènes de pouvoir et de domination), permettant ainsi de rappeler qu'il existe des possibilités alternatives à l'ordre établi (avec une visée d'émancipation et de justice sociale). Une telle posture interdit l'euphémisme scientifique qui masque les émotions violentes derrière des catégories dépassionnées : puisque l'objectif est de déstabiliser le statu quo, un langage précis mais sans compromis est généralement adopté, ce qui a aussi le mérite de ne pas masquer ses propres influences pour faire apparaître une fausse neutralité.

Selon notre analyse des matériaux recueillis, le traitement journalistique de cette «affaire » nous semble correspondre à un scandale «manqué ». En effet, cet événement aurait pu être l'occasion pour les journalistes et les citoyens d'interpeller certains aspects de la gestion étatique contemporaine. On y découvre que les gouvernants de plusieurs pays utilisent des technologies financières pour manipuler l'image comptable des finances publiques, ayant recours à des instruments dont la seule utilité est de réduire le déficit public à court terme mais dont la conséquence est de l'accroître à long terme. Pourtant, les positions critiques, reliant par exemple la «crise grecque» à l'utilisation par les gouvernements de ces technologies de finance créative, disparaissent de l'agenda journalistique en quelques jours. De nouvelles interprétations et mises en accusation, peu susceptibles de déstabiliser le statu $q u o$, les remplacent. Les trajectoires problématisantes suivies par les médias écrits viennent ainsi conforter la tendance néolibérale.

Après avoir présenté un bref panorama des techniques de «finance créative » utilisées par les gouvernements européens pour manipuler l'image comptable des finances de l'État, nous procédons à l'analyse critique des différentes mises en accusation et solutions proposées dans les principaux médias au sujet de la crise grecque pour montrer comment certaines trajectoires problématisantes permettent de vider les «crises» de leur potentiel de déstabilisation du statu quo.

\section{La propagation des techniques de finance créative au sein de l'appareil public}

La crise grecque commence quand son président nouvellement élu annonce fin 2009 que le gouvernement précédent avait, depuis plusieurs années, cherché à «enjoliver » ses comptes, notamment en réduisant l'ampleur de sa dette publique, et avec l'aide d'une grande banque d'affaires établie aux Etats-Unis - Goldman Sachs. Le mécanisme employé, qui n'était pas spécifiquement interdit par Eurostat ${ }^{2}$ à l'époque, repose sur une opération de «swap de devises » (cross currency swap), et aurait permis de faire disparaître (au moins temporairement) un montant d'un milliard d'euros de dette publique ${ }^{3}$, Goldman Sachs

${ }^{2}$ L'Office statistique des Communautés européennes, généralement dénommé «Eurostat», émanation de la Commission Européenne, est chargé de produire les statistiques officielles de l'Union Européenne à partir des statistiques nationales communiquées par ses États membres.

${ }^{3}$ En fait, même après l'opération, la dette grecque reste très supérieure à ce qui est prévu par les critères de convergence. Cependant, entre 2001 et 2010, il y a entre cinq et douze pays sur seize (avec une moyenne supérieure à huit sur seize) qui ne les respectent pas. De plus, 
recevant pour son «expertise » un montant évalué à 200 millions de dollars (Dunbar, 2003). Après avoir présenté un bref panorama des techniques de finance créatives utilisées par les gouvernements européens, nous présentons plusieurs rapports documentant leur propagation et offrant des problématisations antérieures à la crise qui nous intéresse.

\subsection{Panorama des techniques de finance créative}

Les produits dérivés, qui ont connu un développement extrêmement rapide à partir des années 1990 et surtout dans les années 2000, sont souvent présentés comme très complexes mais aussi comme le dernier raffinement de «l'art» des «génies » de la « haute finance ». A l'époque, leur utilité et leur légitimité n'étaient pas réellement remises en question, jusqu'à ce qu'ils soient présentés comme ayant favorisé, voire même déclenché la crise économique et financière internationale qui a débuté en 2007. Il apparaît alors que les produits dérivés peuvent être utilisés pour faire disparaître de la dette, notamment les «junk bonds » au cœur de la crise dite des « subprimes ».

Cependant, les entreprises privées ne sont pas les seules organisations ayant utilisé ces technologies de finance créative pour «gérer leur dette »- les gouvernements le font aussi. En fait, le gouvernement grec n'est même pas le premier à les mobiliser : le gouvernement italien les utilisait depuis 1996 (Dunbar, 2003). Les gouvernements allemand, français et belge ont eux-mêmes eu recours à différents instruments financiers pour diminuer la valeur comptable de leur dette tout en continuant à emprunter (Brown et Chambers, 2005) - ce qui ne les a pas empêchés de dénoncer fortement le « comportement grec ».

Plusieurs techniques de finance créative ont été utilisées par différents gouvernements européens (voir notamment Piga, 2001 ; Brown et Chambers, 2005). Le mécanisme le plus fréquemment employé consiste pour un gouvernement à prendre à sa charge les systèmes de retraites relatifs à des entreprises publiques et à vendre les actifs sous-jacents : le gouvernement obtient des liquidités, et n'inscrit pas comme dette sa responsabilité dans le paiement futur de ces retraites. Une autre technique utilisée repose sur l'externalisation d'une partie de l'action publique, ainsi que du besoin de financement correspondant, vers des entreprises implicitement cautionnées par l'État mais dont les comptes ne sont pas consolidés dans les statistiques publiques : c'est le principe du montage déconsolidant utilisé par Enron. D'autres opérations font intervenir des techniques de titrisation (conversion d'actifs en titres négociables sur un marché), la création d'un impôt remboursé l'année suivante, ou les contrats de swaps de devises. Cette dernière opération est celle utilisée par la Grèce.

Le principe consiste à émettre un emprunt libellé dans une monnaie étrangère, par exemple en dollars, puis à signer un contrat pour échanger les versements en dollars contre des paiements en euros. Or, ce contrat entre un gouvernement et une banque peut prévoir un taux de change différent de celui négocié sur le marché des changes international. Pourtant, c'est ce taux de marché qui était utilisé jusqu'en 2004 dans le calcul du montant de la dette publique communiqué à Eurostat. Si le contrat prévoit un taux particulièrement favorable, le

l'Allemagne (dès 2002) puis la France (dès 2003) cessent elles-mêmes rapidement de respecter ces critères. 
gouvernement obtient plus d'euros, pour chaque dollar emprunté, que ce qu'il comptabilise (en retour, au moment des remboursements, il versera un montant supérieur). Le différentiel constitue donc bien une somme «prêtée » par la banque et remboursée ultérieurement par le gouvernement, un emprunt public dont la particularité est de ne pas apparaître dans les comptes publics. Par exemple, en empruntant 100 dollars puis en négociant un swap à un dollar pour un euro quand le taux officiel est à 1,45 le gouvernement reçoit 100 euros mais n'inscrit que 69 euros en dette ; la différence de 31 euros est la valeur négative du swap et devrait, selon les principes de la logique conservatrice comptable, être comptabilisée comme actif négatif ou comme dette.

Dans toutes ces opérations, il s'agit donc de transférer une partie de la dette publique à d'autres entités ou de comptabiliser des dettes non au passif mais comme des revenus. Ce stratagème est possible parce que les normes comptables européennes n'ont obligé à intégrer les produits dérivés dans le calcul de l'endettement des pays européens qu'à partir de 2004 : jusque-là, les produits dérivés n'apparaissaient qu'au niveau des charges et des revenus financiers, pas au bilan.

\subsection{Problématisations initiales et plaidoyers pour la transparence}

Lorsqu'elles apparaissent sur la scène médiatique, en 2010, ces techniques de financement sont présentées comme des manipulations dont l'objectif est de déformer l'image comptable des dettes publiques: le problème identifié est celui du manque de «transparence ». Pourtant, lesdites techniques font l'objet dès le début des années 2000 de plusieurs rapports, qui en soulignent les risques en matière de gestion de la dette publique. Le FMI et la Banque Mondiale (2001), par exemple, regrettent leur manque de «transparence » mais, pour ces institutions, la «transparence » n'a qu'un seul destinataire: les marchés financiers. Un universitaire italien publie aussi un rapport (Piga, 2001) très détaillé et décrivant des cas précis de gestion de dette publique. Ce dernier dénonce plutôt le manque de communication vis-à-vis des citoyens, et met en cause les règles comptables adoptées par l'Union Européenne. En effet, à partir du moment où des objectifs rigides sont imposés, ce qui est le cas des critères de Maastricht notamment, mais où les normes comptables sont ambiguës, il devient plus facile pour un gouvernement de jouer sur des règles de gestion et de comptabilisation des instruments financiers que de réduire les dépenses publiques ou d'augmenter les impôts.

Les pratiques de finance créative étaient donc répandues et connues par différents acteurs politiques depuis le début des années 2000 sans que le scandale n'éclate. Plusieurs communiqués Eurostat, des rapports d'universitaires, de certaines institutions internationales, de la Banque Centrale Européenne (BCE) et même des articles de revues spécialisées font état de ces pratiques. Piga (2001) va plus loin, puisque selon lui ces pratiques ont été l'enjeu de discussions entre les statisticiens européens, qui souhaitaient réformer les règles comptables européennes, et les gestionnaires de dette publique des pays membres, ces derniers ayant obtenu gain de cause, c'est-à-dire le droit de continuer à "gérer » (l'image comptable de) leur dette en utilisant les produits dérivés. Son argumentation est d'autant plus convaincante que «l'affaire grecque » de 2010 n'est pas la première du genre : en 2004, Eurostat publie un document accusant les gestionnaires de dette grecs d'avoir manipulé les statistiques publiques et réévaluant les montants de la dette grecque publiés entre 1997 et 2003 (Eurostat, 2004). 
Alors, quelques articles paraissent dans la presse mais l'affaire ne fait pas scandale - peut-être parce qu'à l'époque le gouvernement grec n'est pas le seul à pratiquer la finance créative. Au final, le traitement journalistique de cette affaire de manipulation des comptes publics, autant en 2004 qu'en 2010, nous semble correspondre à un scandale "manqué ». Il y avait là l'occasion pour les journalistes et les citoyens d'interpeller certains aspects de la gestion étatique contemporaine : il en fut autrement.

\section{Mise à l'épreuve et trajectoire problématisante}

Nous présentons maintenant la trajectoire problématisante qui a été privilégiée par la presse autour de «l'affaire» grecque au début de l'année 2010. Si diverses voies de problématisation apparaissent initialement dans les énoncés journalistiques, on assiste très rapidement à une certaine homogénéisation des interprétations : en étudiant les déplacements progressifs de la critique formulée par les acteurs politiques et médiatiques, ainsi que les solutions proposées, nous cherchons à comprendre comment l'agenda néolibéral de managérialisation des États s'est comporté face à une mise à l'épreuve dans l'arène sociale.

\subsection{La mise en accusation : d'une critique des spéculateurs à une critique des citoyens grecs}

Au cours des premiers mois de l'année 2010, alors que le gouvernement grec tente de lever des fonds sur les marchés internationaux de capitaux, le taux obligataire auquel les parties offrent d'acheter ces titres s'envole ; ce phénomène est interprété, par les gouvernants comme par les journalistes, comme le signe que les «investisseurs » anticipent un défaut de paiement de l'État grec : la « crise grecque » est ouverte.

Dans un premier temps, la presse s'intéresse au rôle des instruments financiers. Deux types d'instrument sont pointés du doigt: les technologies construites par des banques d'affaires pour aider les gouvernants grecs à maquiller leurs comptes, d'une part, et celles utilisées par les spéculateurs pour se protéger contre (ou parier sur) un risque de défaut de paiement de l'État grec, d'autre part. Ainsi, les produits dérivés, notamment ceux utilisés pour se couvrir contre un risque de défaut de paiement («credit default swaps", CDS), sont accusés de déstabiliser le pays, critique relayée par exemple par le président de la Réserve Fédérale des États-Unis. Certains rappellent alors que ce sont aussi des produits dérivés qui avaient été à l'origine de la crise dite des «subprimes », et la critique se fait plus mordante : «Le fait est que les swaps de couverture de défaillance et autres dérivés complexes qui se sont révélés être des instruments de destruction massive restent bien établis dans notre système financier trois ans après que notre économie a été mise à genoux » (Morgenson, 2010).

Plus précisément, ce n'est pas le fait pour un investisseur de se couvrir contre un risque de crédit qui est dénoncé, mais la possibilité qu'il a d'acheter un tel produit alors même qu'il ne possède aucun titre de la dette concernée. Cette possibilité de se couvrir contre un risque que l'on ne supporte pas est rapprochée de l'interdiction qui existe de souscrire un contrat d'assurance pour une habitation que l'on ne possède pas, puisque cela inciterait à y mettre le feu. La mise en accusation porte alors sur la faible réglementation et l'opacité dont bénéficient les produits dérivés, ainsi que sur le comportement des spéculateurs cherchant à 
nuire à l'économie d'un pays, critiques relayées par différents acteurs politiques. Seul The Economist (2010) prend la défense des investisseurs, en expliquant qu'il serait contreproductif de blâmer « les marchés » :

Les gouvernements ont arrêté de chercher à soutenir les marchés financiers et ont commencé à les rendre responsables de toute la misère du monde. Au lieu d'admettre que l'augmentation des taux obligataires en Europe du sud est une réponse rationnelle à une détérioration de leurs finances publiques, les politiciens croient que ces pays agités ont été injustement attaqués par les spéculateurs. (...) Cependant, il ne semble pas très sage pour les gouvernements européens de commencer à taper sur les gens à qui ils ont besoin d'emprunter des centaines de milliards d'euros.

The Economist présente donc les marchés financiers, non comme déstabilisateurs, mais comme des « sauveurs », allant jusqu'à affirmer que des investisseurs n'appréciant pas d'être critiqués pourraient refuser d'acheter des titres de dette publique, ce qui serait catastrophique pour les États.

Si les autres supports critiquent dans un premier temps le comportement des spéculateurs de manière indifférenciée, on peut progressivement observer une inflexion significative de la trajectoire problématisante suivie en ce que la mise en accusation se focalise progressivement sur un acteur particulier. En effet, comme le font remarquer différents journalistes, l'institution qui a produit les technologies financières de maquillage des comptes publics grecs spécule elle-même contre la solvabilité du pays. Très rapidement, la mise en accusation se focalise alors sur cette banque :

Alors que la Grèce chancèle au bord du chaos fiscal, menaçant d'entraîner avec elle une grande partie de l'Europe, le rôle de Wall Street dans ce fiasco suscite un mépris bien mérité. Au départ on apprend que la Grèce a échangé des produits dérivés avec Goldman Sachs pour cacher sa dette publique. Ensuite on découvre que cette même banque utilise des produits dérivés (...) pour parier sur un défaut de la Grèce et pour spéculer contre l'euro. (The New York Times, 2010b)

Donc Goldman Sachs, la banque d'investissement la plus grande et la plus présomptueuse au monde, a été poursuivie pour fraude par la SEC. Légalement, le cas repose sur une question technique. Moralement, cependant, le cas Goldman Sachs pourrait se transformer en référendum ultime sur l'ethos du bien-fondé de l'avarice («greed is good») qui a conquis l'Amérique dans les années 1980. (Taibbi, 2010)

Alors que la problématisation initiale de la crise grecque concernait avant tout les produits dérivés et le système bancaire et spéculatif de manière générale, elle se focalise progressivement sur une banque particulière. Outre son rôle dans la crise grecque, celle-ci est accusée d'avoir vendu à ses clients des produits financiers avant de spéculer sur leur effondrement, ce qui pourrait avoir accentué la crise dite des subprimes. Or, ces deux «affaires » sont révélées dans un intervalle de quelques semaines : tous les regards se tournent alors vers cette banque, dont la moralité des dirigeants est questionnée. Ces énoncés, qui n'opèrent pas de véritable distinction entre les deux crises, mais les présentent comme deux éléments à charge contre un même acteur, semblent laisser supposer que tous les problèmes issus de la finance créative doivent être imputés, non à la prolifération des produits dérivés, mais à un non-respect des principes déontologiques généralement admis au sein des institutions financières. La mise en accusation se focalise donc sur un acteur particulier et sur 
ses valeurs : le système bancaire peut être préservé, puisque c'est un mauvais joueur qui est en cause.

La problématisation médiatique a donc connu une première inflexion en ne critiquant plus les produits dérivés de manière générale, mais en tant qu'outils pouvant être utilisés par une banque pour contourner les règles déontologiques de la profession. Cependant, à partir de la fin du mois de mars 2010, les médias se désintéressent progressivement de ces instruments abstraits pour se focaliser sur la situation de l'économie grecque ${ }^{4}$. Ainsi, de nombreux journalistes expliquent que l'État grec a vécu au-dessus de ses moyens. Ses dépenses publiques et son système de retraites sont présentés comme particulièrement " généreux »; plus grave, la corruption et l'économie souterraine y seraient « endémiques »:

Le pays a choqué par la faiblesse de ses institutions politiques. Les statistiques économiques officielles se sont révélées mensongères, la corruption est endémique et la collecte des impôts très incomplète - pour le dire gentiment. (...) Certains considèrent qu'il existe de nombreuses possibilités pour resserrer la ceinture. Le système public de retraites, par exemple, est «parmi les plus généreux » au monde, selon l'OCDE. (...) Ce n'est pas uniquement la solvabilité du pays qui focalise l'attention internationale, mais aussi son honnêteté. (Atkins et Hope, 2010)

En regardant les manifestants et les politiciens en Grèce (...), on se demande pourquoi tout ça leur échappe. Ils ont profité d'avantages publics plus généreux que ce qu'ils pouvaient se permettre. Aucune manifestation et aucun fonds de sauvetage n'y changera quoi que ce soit. (...) Les citoyens grecs vont rapidement avoir plus de difficultés à prendre leur retraite à 40 ans. (Leonhardt, 2010)

Il y a peu de doute que la crise de la dette grecque soit de son propre fait : c'est le résultat de la corruption, de la fraude fiscale et de cette séduisante situation méditerranéenne associant un haut niveau de vie et une faible productivité. (Pearlstein, 2010)

Les citoyens grecs sont présentés comme des «tricheurs », cherchant par tous les moyens à travailler le moins possible et à ne pas payer leurs impôts. Le problème ne vient donc pas des instruments financiers, mais des grecs eux-mêmes : ce n'est plus un problème économique, mais «culturel ». Fainéants, voleurs, menteurs : ne correspondant pas à l'idéal du travailleur professionnel, honnête et mobilisé pour aider l'économie de son pays, les citoyens grecs auraient plongé eux-mêmes leur pays dans la crise.

\subsection{Les solutions proposées : un renforcement de la surveillance des États}

La problématisation du lien entre crise financière et comptabilité des États prend un sens particulier lorsque l'on examine les solutions proposées par les gouvernants des autres pays européens - auxquels la presse écrite fait largement écho. Ces «solutions » s'orientent

${ }^{4}$ Nous identifions ici une trajectoire globale sans chercher à recenser tous les articles qui pourraient se distinguer. Par exemple, certaines critiques des banques d'affaires et des produits dérivés paraissent après juin 2010. Cependant, ces articles sont noyés dans un ensemble dont le message global subit un infléchissement au cours du mois de mars 2010. C'est cet infléchissement qui nous permet de comprendre la narration générale qui a entouré la « crise grecque ». 
toutes vers deux éléments - la mise en place de «plans d'austérité » et davantage de surveillance.

Ainsi, pour « remettre en ordre » les comptes du gouvernement grec, plusieurs États européens décident de placer le pays sous une «tutelle budgétaire ». Plus précisément, des « experts » sont chargés d'auditer les comptes grecs. Il s'agit de membres de la BCE et du FMI, mandatés par la Commission Européenne (DGEFA, 2010). Leur mission concerne en fait deux éléments : s'assurer que les comptes publics grecs sont " justes », d'une part, et que le gouvernement adopte des «mesures d'austérité adéquates pour réduire son déficit public », d'autre part. Si le premier élément s'apparente à une mission d'audit externe, en apparence neutre et donc légitime, le second est plus politique et peut être perçu comme une ingérence dans les choix d'un gouvernement souverain. L'argument proposé est lui aussi double. D'une part, plusieurs gouvernements européens, ainsi que le FMI, envisagent d'aider le gouvernement grec, soit par le versement d'un prêt, soit par la garantie de sa dette. D'autre part, il s'agit de restaurer la confiance des marchés des capitaux. Ainsi, le choix est justifié par son caractère objectif, au sens d'extérieur : ce sont les «marchés », acteur abstrait s'il en est, considéré comme indépendant de tout groupement politique, qui se défient de la solvabilité du gouvernement grec, et rendent donc nécessaire une intervention pour «aider à restaurer la confiance ».

Les marchés s'amusent à tester l'euro (...). Mais les marchés ont aussi accru leur pression sur les plus grandes économies européennes, comme l'Allemagne et la France, pour trouver des moyens de sauver la Grèce. (Erlanger, 2010)

La semaine dernière, les leaders politiques européens ont rassuré les marchés obligataires paniqués en affirmant qu'ils s'assureraient - d'une manière ou d'une autre - que la Grèce ne fasse pas défaut. Cette semaine, les ministres des finances ont donné à Athènes un mois pour démontrer que les mesures draconiennes qu'elle a annoncées (...) seront suffisantes pour atteindre l'objectif de réduction du déficit. (The New York Times, 2010a)

La Grèce a promis à ses alliés de la zone euro de diminuer son déficit de $12,7 \%$ à $8,7 \%$ cette année. Bien sûr, et les poules ont des dents. Ou alors appelons Goldman Sachs pour de nouvelles illusions d'optique. (...) Et que se passera-t-il si les dieux (les marchés obligataires) se mettent en colère et refusent de payer? (Garton Ash, 2010)

Dans ces trois extraits, les médias mettent en scène des marchés financiers en position de force par rapport aux gouvernements, comme si la relation de pouvoir était unidirectionnelle. Ces extraits illustrent aussi comment les gouvernements européens, et notamment français et allemand, ont traduit la «crise grecque » en termes de responsabilité des membres du gouvernement grec, impliquant une solution reposant sur l'intervention politique - l'aide est conditionnée à la mise en place, par le gouvernement socialiste, d'une politique conservatrice - et la surveillance des pratiques comptables et financières de l'État. Les solutions envisagées ne concernent donc pas les mécanismes et les logiques qui ont amené un gouvernement à mobiliser des technologies de finance créative, mais reposent sur une discipline qui serait induite par le marché et par certains mécanismes de surveillance. Cet exercice de disciplinarisation est pudiquement caché derrière les notions «d'audit» et de «gouvernance économique », inspirées du vocabulaire des entreprises privées, et présentées comme une évolution heureuse :

À long terme, la crise peut bien avoir rendu la zone euro un peu plus forte grâce à un 
nouvel élément prudemment qualifié de «gouvernance économique ». (Garton Ash, 2010)

La Commission Européenne, en réponse à une crise grecque qui reflète partiellement ses propres faiblesses de supervision, a demandé aux gouvernements européens de soumettre leurs budgets nationaux à un processus de révision avant présentation aux parlements nationaux. «Une révision par les pairs des politiques fiscales pourrait aider l'UE et la zone euro à formaliser une position fiscale globale », selon un communiqué de la Commission. (AFP, 2010)

Ainsi, les gouvernants prévoient de généraliser les technologies de surveillance proposées dans le contexte de la crise grecque. Au départ, les pratiques jugées non éthiques déployées par le gouvernement grec ont permis de légitimer une intervention plus directe de représentants des agences internationales néolibérales dans les choix de politique publique d'un État. Une fois accepté le principe de supervision des politiques publiques par des acteurs ne bénéficiant pas de mandats électifs, il devient possible de généraliser les pratiques de surveillance à l'ensemble des États membres de l'Union Européenne. Certains envisagent même la possibilité de créer un «Fonds Monétaire Européen ». L'objectif est de déployer de nouveaux dispositifs de supervision et de discipline des gouvernements, les choix budgétaires et financiers devant être subordonnés à des institutions supranationales indépendantes des systèmes démocratiques et politiques nationaux. Le néolibéralisme ressort ainsi grandi d'une crise qui aurait pu, en principe, contribuer à affaiblir son emprise sur les pratiques étatiques.

\subsection{Synthèse : notre interprétation de la trajectoire suivie}

Il est maintenant possible de proposer quelques pistes d'interprétation de cette trajectoire problématisante. Dès la fin des années 1990, plusieurs gouvernements européens adoptent des pratiques leur permettant de diminuer la valeur comptable de leur endettement. Au début des années 2000, ces pratiques font l'objet de plusieurs rapports qui aboutissent à des modifications des normes comptables publiques européennes, mais sans déclencher de débat public avant 2010. On assiste alors à la médiatisation de la crise grecque et à un exercice de construction de sens qui, assez rapidement, fait apparaître sur le devant de la scène et place sur le banc des accusés les citoyens grecs et leurs représentants politiques.

Alors que plusieurs axes de problématisation sont initialement développés, ce sont plutôt les stéréotypes concernant les citoyens grecs ${ }^{5}$ et la défiance à l'encontre des comptes publics grecs qui structurent cette nouvelle trajectoire de problématisation. Corrompus, fainéants et tricheurs, les citoyens grecs sont présentés comme des membres de «ces pays du Club Med» bénéficiant de «cette séduisante situation méditerranéenne associant un haut niveau de vie et une faible productivité » (Pearlstein, 2010). Il ne s'agit plus uniquement de réformer les pratiques financières européennes, mais d'améliorer la moralité des citoyens grecs et de leurs représentants, stigmatisation qui sert de justification à l'établissement d'un régime de surveillance économique au sein de l’Union Européenne.

5 Elias et Scotson (1965) montrent bien que les stéréotypes négatifs de l'autre servent généralement à renforcer les stéréotypes positifs de soi (ce qu'ils nomment «l'idéal du nous »). 
La plupart des articles traitant des protestations des citoyens grecs contre les plans d'austérité qui leur sont imposés ne manqueront pas de rappeler que leur gouvernement a «maquillé ses comptes » (sans préciser que d'autres gouvernements européens ont adopté les mêmes pratiques). Ce rappel indigné de la «malhonnêteté » grecque est au cœur de la mise à l'épreuve de l'agenda néolibéral relatif à la managérialisation des États. En effet, la trajectoire problématisante suivie permet d'éviter que «la crise» n'incite au questionnement du néolibéralisme lui-même : en s'orientant vers la dénonciation du comportement déviant de certains acteurs, ce n'est pas un système qui est mis en cause, mais des citoyens et des institutions spécifiques. Le «marché » est invoqué comme principe d'évaluation des politiques publiques, mais permet aussi d'accroître l'influence de certains acteurs politiques : de nouveaux outils de contrôle et de discipline sont introduits de manière à accroître la surveillance de pays «périphériques » par certains acteurs «centraux ». Il n'est ainsi pas anodin que, au moment où «le couple franco-allemand» se rêve en «locomotive de l'Europe », ce soient des journalistes allemands (dans un article publié le 8 février 2010 dans le Spiegel) qui permettent, les premiers, de traduire un problème financier obscur en mobilisation internationale scandalisée, ni que ce soient les membres de la BCE et du FMI (deux organismes alors dirigés par des français ${ }^{6}$ ) qui prennent en main la surveillance comptable d'un État membre. Cette «affaire» sert alors de légitimation à un programme néolibéral reposant sur une gestion budgétaire dite «de rigueur », c'est-à-dire conforme à l'esprit du néolibéralisme.

\section{Discussion et conclusion}

Depuis les années 1980, la «modernisation» des appareils publics a été largement influencée par une forme de mimétisme envers des pratiques apparues dans les entreprises privées et présentées comme universellement efficaces. Ainsi, pour être "performant», un État, un ministère ou une entreprise publique se doit de gérer par les résultats, financiers notamment (Chatelain Ponroy et Sponem, 2008). Cette managérialisation des États doit cependant être replacée dans une tendance plus large : celle du néolibéralisme, qui fait du marché le principe organisateur de l'État, non pour en supprimer l'intervention mais pour l'orienter vers la constitution d'une société d'entrepreneurs placés en situation de concurrence. Cet article s'est intéressé aux mécanismes sociaux qui permettent à la tendance néolibérale de sortir renforcée de ses mises à l'épreuve. Nous avons mis en exergue une trajectoire naturalisante de problématisation, fondée sur un processus de réinterprétation médiatique des crises du néolibéralisme permettant de les vider de leur potentiel déstabilisateur et participant ainsi à la naturalisation du programme néolibéral.

En effet, l'un des principaux enjeux liés à la propagation du néolibéralisme revient à présenter comme un « fait » l'inévitabilité ou, mieux, l'influence nécessairement bénéfique du libre marché en matière d'administration publique - comme si le gouvernement par le marché

${ }^{6}$ En fonction depuis novembre 2011, le successeur au directeur de la BCE n'est ni allemand, ni français, mais était entre 2002 et 2005, soit pendant le maquillage des comptes grecs, viceprésident et directeur général de Goldman Sachs. 
était une rationalisation incontournable, irrésistible et hautement souhaitable. Évidemment, nous ne nions pas l'existence de discours critiques à l'égard de l'agenda néolibéral de managérialisation des États. Toutefois, notre analyse permet d'identifier une tendance forte, au sein des médias, qui décourage tout questionnement du bien-fondé du managérialisme comme technologie de gouvernement.

Ainsi, aux débuts de la crise financière mondiale, notamment lors de la débâcle de la firme Lehman Brothers, certains médias ont parlé de «crise du capitalisme financier », c'està-dire d'une défaillance du programme néolibéral. On aurait alors pu imaginer, comme l'ont fait de nombreux commentateurs, que cette crise allait déclencher un revirement politique (Morgan et al., 2011). Cependant, la critique a été rapidement orientée vers les banques d'affaires, ce qui a permis de ne pas questionner trop fortement les politiques publiques anglo-américaines ayant favorisé l'endettement des ménages et la spéculation, deux phénomènes au cœur de la crise dite «des subprimes» (McSweeney, 2009). Ensuite, la critique des banques a fait place à la critique des États, sommés de réduire les dépenses publiques sous peine d'être « sanctionnés » par les marchés financiers (Morgan et al., 2011).

De la même manière, les trajectoires de problématisation suivies lors de la «crise grecque » permettent d'éviter une remise en cause des principes, dispositifs et mentalités du programme néolibéral en focalisant l'accusation sur le comportement d'acteurs déviants - une banque, un gouvernement, une nation. En particulier, présentés comme peu travailleurs, ne payant pas leurs impôts, et cherchant toujours «la magouille » pour réussir sans effort, les citoyens grecs n'ont pas intériorisé les valeurs du capitalisme managérial. Cette réinterprétation est cohérente avec le discours néolibéral, selon lequel chacun doit organiser sa vie comme une série d'investissements : les individus sont seuls responsables de leurs échecs qui résultent, nécessairement, d'un refus de se comporter en bon entrepreneur capitaliste (Foucault, 2004).

Ces trajectoires ont détourné l'attention du fonctionnement des marchés financiers ou des instruments qui y ont été fabriqués, ainsi que des relations nouées entre les représentants des banques privées et ceux des gouvernements. L'identification d'un nombre restreint de «transgresseurs » permet aussi de réaffirmer la validité des règles antérieures (alors que certaines règles pouvaient sembler si peu suivies qu'elles en paraissaient facultatives, la mise en accusation revient à désigner certains actes comme transgressifs, donc à présenter les règles antérieures comme de nouveau légitimes, et au passage à faire oublier que l'on a pu soi-même transgresser ces normes par le passé) et de démontrer que le fonctionnement actuel permet d'isoler les mauvais joueurs et donc de renforcer le statu quo, ou plutôt en l'occurrence de maintenir la tendance néolibérale. Les stratégies de mise en accusation, déployées au sein des médias, ont donc permis de limiter les effets déstabilisateurs de la crise sur la tendance néolibérale de managérialisation des États. Le programme néolibéral est préservé, voire renforcé, par cette mise à l'épreuve.

$\mathrm{Au}$ passage, l'indignation médiatique crée une apparence de débat, mais un débat orienté selon une trajectoire qui ne remet pas en cause le néolibéralisme. Nos résultats permettent donc de confirmer l'existence d'un espace pour les narrations critiques du managérialisme (Parker, 2002), mais cet espace a plutôt pour effet de détourner l'attention, de maintenir l'illusion du pluralisme et du débat tout en aboutissant au final à une mise au silence des discours alternatifs en les submergeant de représentations stéréotypées (Said, 2004). Cette trajectoire est d'autant plus facilement suivie que les narrations critiques doivent 
prendre appui sur le fonctionnement de technologies financières complexes, peu transparentes, et par là-même particulièrement difficiles à déconstruire et à questionner : il devient de moins en moins probable que les journalistes et les citoyens saisissent la portée et les conséquences des choix de financement de l'action publique réalisés en leur nom. La prolifération de ces instruments, construits à des fins de spéculation boursière mais présentés comme une «rationalisation» de la gestion financière, explique selon nous en partie le silence des citoyens devant un événement qui aurait pu se transformer en scandale propre à déstabiliser les pratiques établies.

Cette évolution conduit à déconnecter les choix opérés par les gouvernements de tout débat véritablement démocratique. Les décisions les plus politiques sont présentées comme indiscutables, non pas parce qu'elles seraient issues d'un consensus démocratique, mais parce qu'elles reposeraient sur l'utilisation rationnelle d'instruments ayant l'apparence de l'objectivité, de la neutralité et de l'universalité. Nous observons donc un renversement de la problématique démocratique. Les projets d'action gouvernementale ne sont plus perçus comme devant refléter la volonté des citoyens, mais sont contraints, dans une large mesure, de se conformer à la volonté des acteurs financiers et aux principes du néolibéralisme : la politique est réduite à du management (Lascoumes et Le Galès, 2004) et toute idéologie sousjacente est rendue invisible et indiscutable. Les comportements perçus comme incompatibles avec le projet néolibéral sont dénoncés, qu'ils soient ou non majoritaires, et les citoyens exhortés à intérioriser les valeurs, priorités et objectifs dictés par la rationalité gestionnaire.

La principale contribution de notre étude est de montrer que l'idéal démocratique est menacé par une analyse collective et médiatique qui, par sa réorientation des problématisations dans un sens favorable à l'ordre établi, tend à réduire au silence toute pensée critique. Ce scandale manqué illustre l'intérêt d'une démarche critique en sciences de gestion. Les compétences des chercheurs en gestion deviennent nécessaires pour donner aux citoyens les moyens de comprendre les techniques utilisées par les gouvernements et donc de construire un débat public sur des bases plus solides et éclairées.

\section{Bibliographie}

AFP. (2010). Europe takes steps to tame debt crisis. Agence France-Presse, 12 mai.

Allard-Poesi, F., Loilier, T. (2009). Qu'est-ce que la critique en Sciences du Management? Que pourrait-elle être ? Economies et Sociétés 43 (12): 1975-1999.

Atkins, R., Hope, K. (2010). A Heraclean task. Financial Times, 6 mai.

Bourdieu, P. (1998a). L'essence du néolibéralisme. Le Monde Diplomatique 528: 3.

Broadbent, J., Guthrie, J. (1992). Changes in the Public Sector: A Review of Recent "Alternative" Accounting Research. Accounting, Auditing \& Accountability Journal 5 (2): 3-31.

Brown, M., Chambers, A. (2005). How Europe's governments have enronized their debts. Euromoney, $1^{\text {er }}$ septembre.

Chatelain Ponroy, S., Sponem, S. (2008). Comme l'entreprise, l'Etat doit adopter une "culture du résultat." In Petit bréviaire des idées reçues en management (Eds, Pezet, A., Sponem, S.). Paris: Editions La Découverte, 257-267.

Dardot, P., Laval, C. (2009). La nouvelle raison du monde. Essai sur la société néolibérale. Paris: Editions La Découverte. 
Directorate-General for Economic and Financial Affairs (DGEFA) (2010). The Economic Adjustment Programme for Greece. European Commission.

Duménil, G., Lévy, D. (2000). Crise et sortie de crise. Ordre et désordres néolibéraux. Paris: Presses Universitaires de France.

Dunbar, N. (2003). Goldman Sachs' mega-deal for Greece. Risk Magazine, 1er juillet.

Elias, N., Scotson, J. L. (1965). Logiques de l'exclusion. Enquête sociologique au cœur des problèmes d'une communauté. Paris: Fayard.

Erlanger, S. (2010). Euro debt crisis is political test. The New York Times, 6 février.

Eurostat. (2004). Report by Eurostat on the revision of the Greek government deficit and debt figures, 22 novembre.

FMI, Banque Mondiale (2001). Guidelines for Public Debt Management, International Monetary Fund and the World Bank.

Foucault, M. (2004). Naissance de la biopolitique. Cours au Collège de France (1978-1979). Paris: Gallimard/Seuil.

Fournier, V., Grey, C. (2000). At the critical moment: Conditions and prospects for critical management studies. Human relations 53 (1): 7-32.

Garton Ash, T. (2010). The agonies of the eurozone reflect a far more significant hidden deficit: The spirit that once led Europeans into union has vanished, just as we now face the euro's widely predicted flaws. The Guardian, 24 février.

Golsorkhi, D., Huault, I., Leca, B. (Eds.). (2009). Les études critiques en management. Une perspective française. Paris: Les Presses de l'Université Laval.

Guthrie, J., Humphrey, C., Olson, O., Jones, L. (Eds.) (2005). International public financial management reform: Progress, contradictions and challenges. InformationAge Press.

Harvey, D. (2005). A Brief History of Neoliberalism. Oxford: Oxford University Press.

Hood, C. (1995). The "new public management" in the 1980s: Variations on a theme. Accounting, Organizations and Society 20 (2/3): 93-109.

Lascoumes, P., Le Galès, P. (Eds.). (2004). Gouverner par les instruments. Paris: Presses de la Fondation Nationale de Sciences Politiques.

Leonhardt, D. (2010). Greece, debt and a lesson for the U.S. International Herald Tribune, 13 mai.

McSweeney, B. (2009). The role of financial asset market failure denial and the economic crisis: Reflections on accounting and financial theories and practices. Accounting, Organizations and Society 34: 835-848.

Morgan, G., Froud, J., Quack, S., Schneiberg, M. (2011). Capitalism in crisis: Organizational perspectives. Organization 18 (2): 147-152.

Morgenson, G. (2010). It's Time for Swaps to Lose Their Swagger. The New York Times, 28 février.

Parker, M. (2002). Against Management. Organization in the Age of Managerialism. Cambridge: Polity Press.

Pearlstein, S. (2010). Greek crisis exposes cracks in Europe's foundation. The Washington Post, 7 mai.

Piga, G. (2001). Derivatives and Public Debt Management. Zurich: International Securities Market Association.

Said, E. W. (2004). Humanism and Democratic Criticism. New York: Columbia University Press.

Soederberg, S. (2008). A Critique of the Diagnosis and Cure for "Enronitis": The Sarbanes-Oxley Act and Neoliberal Governance of Corporate America. Critical Sociology 34 (5): 657-680.

Tabb, W. K. (2003). After Neoliberalism? Monthly Review 55 (2): 25-33.

Taibbi, M. (2010). Will Goldman Sachs prove that greed is God? The Guardian, 24 avril. 
The Economist (2010). Rescuing the rescuers. The Economist, 27 mai.

The New York Times (2010a). Greece Inches Back. The New York Times, 19 février.

The New York Times (2010b). A.I.G., Greece, and Who's Next?. The New York Times, 5 mars. 